\title{
Comments on the kinematical structure of loop quantum cosmology
}

\author{
J. M. Velhinho \\ Departamento de Física, Universidade da Beira Interior \\ R. Marquês D'Ávila e Bolama, 6201-001 Covilhã, Portugal \\ jvelhi@dfisica.ubi.pt
}

\begin{abstract}
We comment on the presence of spurious observables and on a subtle violation of irreducibility in loop quantum cosmology.
\end{abstract}

\section{Introduction}

Loop quantum cosmology (LQC) originated from the works of M. Bojowald (see e.g. [BMT] and references therein) and was later on reformulated in [ABL]. It is an application to quantum cosmology of methods and ideas adapted from those used in loop quantum gravity ${ }^{1}$ (LQG). This adaptation of LQG methods to the quantization of classical cosmological models produced spectacular results (see e.g. [B1, BMT]). However, foundational and conceptual issues are still open.

LQC is in several aspects quite different from LQG, and presents difficulties that are absent in LQG. These are mainly due to the lack of diffeomorphism invariance, which plays a crucial role in the very construction and methods of LQG.

An important open question is the appearance of a large surplus of solutions of the LQC Hamiltonian constraint [ABL, B1]. Partly related to this is the nonseparability of the kinematical Hilbert space, i.e. it does not possess

\footnotetext{
${ }^{1}$ For reviews of loop quantum gravity see $[\mathrm{T} 1, \mathrm{R}, \mathrm{AL}]$.
} 
a countable basis. (Here kinematical means that the Hamiltonian constraint is still to be imposed.) Nonseparability, eventhough at the kinematical level, is an odd feature in quantum mechanics. Moreover, there are increasing indications that even in full LQG a separable Hilbert space is achieved prior to imposing the Hamiltonian constraint [Z, R, T2, V, FR].

We will try to show in the present letter that nonseparability is not mandatory in LQC. Moreover, it is our understanding that this question is surpassed in importance by two intrinsic aspects of the current LQC formulation. The first question concerns a subtle violation of irreducibility. The second is an unexpected commutation involving the quantum Hamiltonian, which is responsible for the appearance of spurious observables, and therefore for the excess of solutions of the Hamiltonian constraint. Both questions are related to the particular form of the LQC Hamiltonian constraint operator.

We assume in what follows that the reader is familiar with the current formulation of LQC as given in [ABL] (see also [AL]), which we take as our starting point. Only a minimal summary of the necessary kinematical setup is included here. As in $[\mathrm{ABL}]$, we restrict ourselves to the simplest situation of spatially flat, homogeneous and isotropic cosmological models.

\section{Kinematical setup}

The classical gravitational phase space that results from the connection variables formalism is coordinatized by real variables $c$ and $p$, with canonical Poisson bracket

$$
\{c, p\}=8 \pi \gamma G / 3,
$$

where $\gamma$ is the Barbero-Immirzi parameter. The variable $p$ has dimensions of (lenght) $)^{2}$ and is directly related to the scale factor $a,|p|=a^{2}$. The connection related variable $c$ is dimensionless. There remains a single constraint, namely the Hamiltonian constraint:

$$
-\frac{6}{\gamma^{2}} c^{2} \operatorname{sgn}(p)|p|^{1 / 2}+8 \pi G C_{\text {matter }}=0
$$

where sgn is the sign function and $C_{\text {matter }}$ is the matter Hamiltonian.

The kinematical variables chosen in $[\mathrm{ABL}]$ to quantize (the gravitational part of) this system consist of $p$ and the commutative algebra $\mathcal{C}$ of almost periodic functions of $c$, whose elements are finite linear combinations of exponentials $e^{i \mu c / 2}$, where $\mu$ can take any real value. The algebra $\mathcal{C}$ clearly 
separates points on $\mathbb{R}$. Together with $p$, we have a Poisson algebra, with

$$
\left\{e^{i \mu c / 2}, p\right\}=\frac{8 i \pi \gamma G \mu}{6} e^{i \mu c / 2},
$$

which is complete, i.e. it separates points in phase space.

The LQC quantization proceeds as follows. Let $\mathcal{H}$ be the Hilbert space spanned by mutually orthogonal vectors $|\mu\rangle, \mu \in \mathbb{R},\left\langle\mu^{\prime} \mid \mu\right\rangle=\delta_{\mu^{\prime} \mu}$, where $\delta_{\mu^{\prime} \mu}$ is the Kronecker delta (not the Dirac distribution). By construction, $\mathcal{H}$ is nonseparable. The quantum operators $\hat{p}$ and $e^{\widehat{i \mu c} / 2}$ are defined by

$$
\hat{p}|\mu\rangle=\frac{8 \pi \gamma G \hbar}{6} \mu|\mu\rangle=\frac{\gamma \ell_{P}^{2}}{6} \mu|\mu\rangle,
$$

where $\ell_{P}=(8 \pi G \hbar)^{1 / 2}$ is the Planck lenght, and

$$
e^{\widehat{i \mu^{\prime} c} / 2}|\mu\rangle=\left|\mu+\mu^{\prime}\right\rangle \text {. }
$$

The commutation relations

$$
\left[e^{\widehat{i \mu c / 2}}, \hat{p}\right]=i \hbar \frac{8 i \pi \gamma G \mu}{6} e^{\widehat{i \mu c / 2}}
$$

are satisfied and the representation is irreducible.

Let us consider the gravitational part of the Hamiltonian:

$$
C_{\text {grav }}=-\frac{6}{\gamma^{2}} c^{2} \operatorname{sgn}(p)|p|^{1 / 2} .
$$

The LQC quantum Hamiltonian constraint operator $\hat{C}_{\text {grav }}$ is given by:

$$
\hat{C}_{\text {grav }}|\mu\rangle=3\left(\gamma^{3} \mu_{0}^{3} \ell_{P}^{2}\right)^{-1}\left(V_{\mu+\mu_{0}}-V_{\mu-\mu_{0}}\right)\left(\left|\mu+4 \mu_{0}\right\rangle+\left|\mu-4 \mu_{0}\right\rangle-2|\mu\rangle\right) \text {, }
$$

where $V_{\mu}:=(\gamma|\mu| / 6)^{3 / 2} \ell_{p}^{3}$ and $\mu_{0}$ is a regularization parameter. Effectively, $\mu_{0}$ parametrizes a quantization ambiguity, as the regulator cannot be removed. The currently adopted viewpoint [ABL, AL] is that this ambiguity can be naturally removed by invoking results from LQG and that (8), with the parameter set to $\mu_{0}=\sqrt{3} / 4$, is the correct Hamiltonian constraint operator.

The kinematical setup is completed with the construction of the quantum inverse scale factor (the quantum scale factor being already determined by $\hat{p})$. The explicit form of this operator is not needed in what follows; it is sufficient to say that, like the quantum scale factor, the quantum inverse scale factor commutes with $\hat{p}$, and therefore its eigenvectors are the basis vectors $|\mu\rangle$. 


\section{Reducibility}

Here, and throughout this work, we consider only representations such that zero belongs to the spectrum of $\hat{p}$.

Let us define $\lambda_{k}:=\frac{4 \mu_{0}}{k+1}, k=0,1,2, \ldots$, and let $\xi$ be a real number such that $\mu_{0} / \xi$ is irrational. Let $\mathcal{C}\left(\lambda_{k}, \xi\right)$ denote the subalgebra of $\mathcal{C}$ generated by the two exponentials $e^{i \lambda_{k} c / 2}$ and $e^{i \xi c / 2}$,

$$
\mathcal{C}\left(\lambda_{k}, \xi\right):=\left\{\sum_{n, m \in \mathbb{Z}} a_{n m} e^{\frac{i}{2}\left(n \lambda_{k}+m \xi\right) c}\right\} .
$$

Since $\lambda_{k} / \xi$ is irrational, it is clear that $\mathcal{C}\left(\lambda_{k}, \xi\right)$ separates points on $\mathbb{R}$. Thus, the Poisson subalgebras defined by $p$ and any given algebra $\mathcal{C}\left(\lambda_{k}, \xi\right)$ are classically complete. Moreover, every algebra $\mathcal{C}\left(\lambda_{k}, \xi\right)$ contains proper subalgebras of the same type, namely $\mathcal{C}\left(\lambda_{k}, N \xi\right)$, where $N$ is a nonzero integer. The corresponding Poisson subalgebras are therefore also complete. All these Poisson algebras are, of course, unambiguously quantized in the Hilbert space $\mathcal{H}$.

Let $\mathcal{H}_{k}^{\xi}$ be the closed subspace of $\mathcal{H}$ spanned by vectors of the form $\left|n \lambda_{k}+m \xi\right\rangle$, where $n, m \in \mathbb{Z}$. Each space $\mathcal{H}_{k}^{\xi}$ is obviously a separable proper subspace of $\mathcal{H}$. Although $\mathcal{H}_{k}^{\xi}$ does not carry a representation of $\mathcal{C}$, it carries a (Dirac) representation of the kinematical algebra defined by $\mathcal{C}\left(\lambda_{k}, \xi\right)$ and $p$. We have thus found a complete Poisson algebra, in fact a large family of them, that is quantized but whose action on $\mathcal{H}$ is not irreducible.

In the usual quantization of unconstrained systems the latter conclusion would be, we believe, a clear indication that the representation space $\mathcal{H}$ is too big (see a discussion in this respect in $[G]$ ). In the presence of constraints the question becomes more subtle, as one has to make sure that the quantum constraint operators are not lost when trying to reduce the representation.

In the present case, it is obvious that the only constraint operator, namely $\hat{C}_{\text {grav }}(8)$, has an action on every space $\mathcal{H}_{k}^{\xi}$. The quantum scale factor and the quantum inverse scale factor operators act within $\mathcal{H}_{k}^{\xi}$ as well. Thus, a complete kinematical setup descends from $\mathcal{H}$ to each of the separable spaces $\mathcal{H}_{k}^{\xi}$. We see no reason to work with the full algebra $\mathcal{C}$, and are therefore led to conclude that the space $\mathcal{H}$ is unnecessarily big. Moreover, for $\lambda_{k}$ of the form $\mu_{0} / n$, i.e. for $k+1=4 n, n \in \mathbb{N}$, one can forget $\mathcal{H}$ altogether, start from $\mathcal{H}_{k}^{\xi}$, and reproduce step by step the whole quantization process described in [ABL]. Special values of $k$ are required for this purpose since, for instance, the construction of the quantum Hamiltonian uses the operator $e^{i \mu_{0} c} / 2$, which 
is not defined in $\mathcal{H}_{k}^{\xi}$ for arbitrary $k$.

It turns out that the "obvious" solution to the above reducibility problem, and simultaneously to the separability issue, namely to drop $\mathcal{H}$ and continue in some space $\mathcal{H}_{k}^{\xi}$, is not at all straightforward. Besides ambiguity in the choice of such a space, a major concern is that every such representation is again affected by reducibility. As mentioned above, the Poisson algebra associated to $\mathcal{C}\left(\lambda_{k}, N \xi\right) \subset \mathcal{C}\left(\lambda_{k}, \xi\right)$ is still complete. Thus, one should consider the proper subspace $\mathcal{H}_{k}^{N \xi} \subset \mathcal{H}_{k}^{\xi}$ and reduce the representation again. It is clear that there is no end to this process.

\section{Spurious observables}

A known open question in the current LQC formulation is the appearance of an uncountable surplus of solutions of the quantum Hamiltonian constraint [ABL, B1]. This is obviously reduced to an infinite countable set if one of the $\mathcal{H}_{k}^{\xi}$ representations is used, but the problem remains essentially the same.

The space of solutions is the (generalized) eigenspace of the Hamiltonian operator corresponding to the (generalized) eigenvalue zero. The dimensionality of this space is dictated by the set of operators commuting with the quantum Hamiltonian, i.e. the set of observables. The appearance of spurious solutions therefore reflects problems with the representation of true observables, or the presence of spurious observables, i.e. operators that commute with the quantum Hamiltonian but actually should not. We believe that spurious observables are present in LQC.

In fact, it is trivial to check that $\hat{C}_{\text {grav }}(8)$ commutes with the operator

$$
V\left(4 \mu_{0}\right):=\exp \left(\frac{6 i}{\gamma \ell_{p}^{2}} \frac{2 \pi}{4 \mu_{0}} \hat{p}\right)
$$

(and with $\left.\left(V\left(4 \mu_{0}\right)\right)^{N}, \forall N \in \mathbb{Z}\right)$. This result remains true after inclusion of the matter Hamiltonian, since, apart from matter degrees of freedom, it contains only the quantum scale factor and the quantum inverse scale factor, and both these operators commute with $\hat{p}$. The former commutation apparently has no classical correspondence. Moreover, if one removes by hand the degeneracy in the solution space corresponding to the operator $V\left(4 \mu_{0}\right)$, namely by selecting the subspace where the (dual of the) operator acts trivially (i.e. as the identity operator), one recovers exactly the expected number 
of solutions in the gravitational sector, namely two (in the flat, homogeneous and isotropic case under consideration).

\section{Final remarks}

Both the reducibility issue and the appearance of spurious observables, and therefore the surplus of solutions of the Hamiltonian constraint, are due to the presence of the regulator $\mu_{0}$ in $\hat{C}_{\text {grav }}(8)$. Unless the ambiguity in the quantization of the Hamiltonian is handled in a totally different way, the appearance of such a fixed parameter seems unavoidable. Moreover, in the currently accepted point of view, the parameter $\mu_{0}$ is actually fundamental, i.e. it is determined by the fundamental theory, namely LQG, that expectedly supports the effective LQC approach.

How can one then interpret the difficulties created by the special form of the LQC quantum Hamiltonian? One possible interpretation, obviously biased by Bojowald's original approach, is to accept that the operator $V\left(4 \mu_{0}\right)$, or the operator $V\left(\mu_{0}\right):=\left(V\left(4 \mu_{0}\right)\right)^{4}$, has no meaning whatsoever, not even kinematically. Although radical, this hypothesis finds some support in the

form of $\hat{C}_{\text {grav }}$ itself. One such formal indication is that the spectrum of $\hat{p}$ is representation-dependent, the only invariant feature being precisely the spectral points of the form $4 n \mu_{0} \gamma \ell_{P}^{2} / 6$, or $n \mu_{0} \gamma \ell_{P}^{2} / 6$, if one restricts attention to spaces $\mathcal{H}_{k}^{\xi}$ with $k+1=4 m$.

The above attitude naturally leads to a strong condition on the quantum kinematical Hilbert space, namely that the action of the operator $V\left(4 \mu_{0}\right)$, or $V\left(\mu_{0}\right)$, should be trivial. Trivialization of the operator $V\left(\mu_{0}\right)$ leads to the kinematical Hilbert space used in [B2]. Trivialization of $V\left(4 \mu_{0}\right)$ leads to a subspace of the later space, where the remaining four-fold degeneracy is also removed. In this case, however, one would still have to rely on the bigger space in order to construct the quantum Hamiltonian.

\section{Acknowledgements}

It is a pleasure to thank José Mourão and Thomas Thiemann. This work was supported in part by POCTI/33943/MAT/2000, CERN/P/FIS/43171/2001 and POCTI/FNU/49529/2002. 


\section{References}

[ABL] A. Ashtekar, M. Bojowald, J. Lewandowski, Adv. Theor. Math. Phys. 7, 233 (2003), gr-qc/0304074.

[AL] A. Ashtekar, J. Lewandowski, Background Independent Quantum Gravity: A Status Report, gr-qc/0404018.

[B1] M. Bojowald, Loop Quantum Cosmology: Recent Progress, grqc/0402053.

[B2] M. Bojowald, Class. Quant. Grav. 19, 2717 (2002), grqc/0202077.

[BMT] M. Bojowald, H. Morales-Técotl, Cosmological applications of loop quantum gravity, gr-qc/0306008.

[FR] W. Fairbairn, C. Rovelli, Separable Hilbert space in Loop Quantum Gravity, gr-qc/0403047.

[G] M. Gotay, Obstructions to Quantization, in From Mechanics to Computation (Essays in Honor of Juan-Carlos Simo), J. Nonlinear Sci. Editors (Springer, New York), 271-316 (2000), math$\mathrm{ph} / 9809011$.

[R] C. Rovelli, Quantum Gravity (Cambridge University Press, Cambridge, 2004), at press.

[T1] T. Thiemann, Modern Canonical Quantum General Relativity (Cambridge University Press, Cambridge, 2004), at press, grqc/0110034.

[T2] T. Thiemann, The Phoenix Project: Master Constraint Programme for Loop Quantum Gravity, gr-qc/0305080.

[V] J.M. Velhinho, On the structure of the space of generalized connections, to appear in Int. J. Geom. Meth. Mod. Phys., math$\mathrm{ph} / 0402060$.

[Z] J. Zapata, Gen. Rel. Grav. 30, 1229 (1998), gr-qc/9703038. 